\title{
Analyse des Tabakes und seiner Fabrikate.
}

Fon

\section{Dr. Viktor Vedrödi.}

Die grosse volkswirthschaftliche Bedeutung, welche der Tabak für das Königreich Ungarn besitzt, gab mir Veranlassung, mich mit diesem. Thema zu beschäftigen. Laut den statistischen Ausweisen des königlich ungarischen Ministeriums für Finanzen wurden im Jahre 1892 als Ernte des Jahres $189164,462,929 \mathrm{~kg}$ Tabak um den Preis von 12,195,177 fl. $82 \mathrm{kr}$. österreichischer Währung eingelöst und 19,989,454 $\mathrm{kg}$ Rohtabak im Betrage von $3,739,078$ fl. 47 kr. nach den österreichischen Erbländern exportirt. Von den Fabrikaten wurden in's Ausland exportirt $62,712 \mathrm{~kg}$ (315 Fässer) Virginia- und Kentuckylaugen-Auszug im Betrage von 30,043 fl. $03 \mathrm{kr}$. und ausserdem Rauchtabak, Cigarren und Cigaretten im Betrage von 998 fl. $45 \mathrm{kr}$.

Um die nationalökonomische Bedeutung des Tabakes in landwirthschaftlicher Beziehung zu kennzeichnen, genügt es hervorzuheben, dass laut obbenannten statistischen Ausweisen auf je einem österreichischen Katastral-Joch $(0,5756$ Hectar) im Durchschnitte $801 \mathrm{~kg}$ Tabak geerntet und dafür dem Producenten von Seiten des Finanz-Aerares 148 fl. 19 kr. ausbezahlt wurden.

Die ungarische Naturforscher-Gesellschaft sah sich schon in den siebziger Jahren veranlasst für das Studium dèr ungarischen Tabake einen höheren Preis auszusetzen, welcher auch seiner Zeit Herrn Dr. Thomas Kosutány, Professor der Chemie an der königlichen ungarischen landwirthschaftlichen Academie in Jngarisch-Altenburg für sein »Magyarország jellemzöbb dohànyainak vizsgálata betiteltes Werk zuerkannt und ausgefolgt wurde. Kosutány veröffentlichte in diesem seinem Werke die Analyse von 144 ungarischen Tabakssorten, die sämmt- 
lich auf ihren Gehalt an Ammoniak, Nicotin, Asche und Kalisalpeter untersucht wurden.

Bei Untersuchung auf Ammon und Nicotin benutzte er seine eigene Methode, die in kurzem Auszuge in dieser Zeitschrift 21, 68 durch Kissling mitgetheilt wurde. Ich muss diese Methode hier deshalb ausführlich wiedergeben, weil ich sie im Nachfolgenden eingehender besprechen und kritisch beleuchten will.

Kosutány bestimmte den Ammongehalt dadurch, dass er von dem gut getrockneten und pulverisirten Tabak $20 \mathrm{~g}$ in einer Reibschale mit Wasser befeuchtete und mit überschüssiger Kalkmilch mischte.

Das Gemisch wurde zur Bindung des hierbei frei werdenden Ammoniaks unter eine gut schliessende Glasglocke gestellt, in welche gleichzeitig eine kleine $20 \mathrm{cc}$ Normalschwefelsäure enthaltende Schale eingestellt wurde. Nach $3-4$ tägigem Stehen war das Ammoniak vollständig: absorbirt und es konnte die Schwefelsäure titrirt werden. Er verdünnte die $20 c c$ Schwefelsäure, welche das Ammoniak bereits absorbirt hatten, auf $200 c c$ und benutzte hiervon je $20 c c$ zur Titrirung mittelst Barythydrats.

Die aus dem Tabake frei gewordene Ammonmenge wurde aus der Differenz berechnet.

Den nach der Ammonbestimmung zurïckbleibenden Tabaksrückstand benutzte Kosutány zur Nicotinbestimmung, indem er denselben auf einem Filter 6-7 mal mit je $80-100 c c$ kaltem Wasser auswusch, wobei darauf geachtet wurde, dass man auf das Filter immer nur dann frisches Wasser aufgoss, wenn der vorherige Aufguss bereits ganz herabgetröpfelt war. Die auf diese Weise erhaltene alkalische Lösung wurde in einem Mischcylinder mit 20-25 Volumprocent Petroleumäther unter öfterem Schütteln 24 Stunden hindurch stehen gelassen, nach 24 stündigem Stehen die alkalische Tabakslösung mittelst eines Scheidetrichters von der Petroleumäther-Lösung getrennt und die alkalische Tabakslösung auf die eben beschriebene Weise noch zweimal mit Petroleumäther behandelt.

Zur Bestimmung des in denselben übergegangenen Nicotins wurde der Petroleumäther mit $1 / 10$-Normalschwefelsäure bebandelt, bis er saver reagirte, und unter öfterem Umschütteln $1 / 2$ Tag hindurch stehen gelassen. Die sich hierbei am Boden ansammelnde wässrige Flüssigkeit wurde mittelst eines Scheidetrichters vom Petroleumäther getrennt und letzterer mit erneuerten Mengen Wassers so lange gemischt und geschüttelt, als hinzugetröpfelte Lackmustinctur noch saure Reaction an- 
zeigte. In der auf diese Weise erhaltenen schwefelsauren Flüssigkeit wurde der Ueberschuss an Schwefelsäure mittelst Barythydratlösung zurücktitrirt und aus dem Ergebnisse der Nicotingehalt berechnet.

Unter den Arbeiten über Tabakanalyse, welche in neuerer Zeit veröffentlicht wurden, ist diejenige $K$ issling's (diese Zeitschrift 21, 64) wegen ihrer Vielseitigkeit und Gründlichkeit in erster Reihe beachtenswerth. Wir finden in Kissling's Arbeit die verschiedenen Methoden der Nicotinbestimmung neben einander gestellt und kritisch beleuchtet. Von meinem Standpunkte aus muss ich jedoch bedauern, dass er die Skalweit'sche Methode weitläufig erörtert und einer gründlichen Kritik unterwirft, Kosutány's Methode dagegen, die doch so vielen wichtigen Tabakanalysen als Grundlage dient, nur nebensächlich erwähnt und einer eingehenden Besprechung gar nicht würdigt, so dass man - obgleich dieses nicht klar ausgesprochen ist - geneigt sein könnte, sie als ganz unzuverlässig und unbrauchbar anzusehen.

Die vielseitigen und gründlichen Versuche, welche Kissling zur Begründung seiner eigenen Methode ausführte, erweckten mein Vertrauen und bestimmten mich, meine Analysen nach seiner Methode auszuführen. Um aber auch über Kosutány's Methode ein klares Bild zu erhalten, machte ich mit einer jeden Tabakssorte Parallelversuche nach seiner Methode; es war dieses eine Pflicht, die ich zufolge meiner amtlichen Stellung seinen analytischen Arbeiten und seiner Persönlichkeit gegenüber in collegialer Hinsicht schuldete.

Bekanntlich bestimmt Kissling den Nicotingehalt dadurch, dass er eine gewisse Menge des Tabakes bei $50-60^{\circ} \mathrm{C}$. trocknet, pulvert und von der pulverigen Masse $20 \mathrm{~g}$ in einer Reibschale mit $10 \mathrm{cc}$ verdünnter alkoholischer Natronlösung (6 $g$ Natronhydrat in $40 \mathrm{cc}$ Wasser gelöst und mit $60 \mathrm{cc} 95$ procentigen Weingeistes gemischt) sorgfältig imprägnirt. Das so erhaitene mässig feuchte Pulver wird in eine passende Hülse aus Fliesspapier geschüttet und im Extractionsapparate mit Aether extrahirt. Die Extraction nimmt 2-3 Stunden in Anspruch. Der Aether wird jetzt behutsam bis auf einen kleinen Rest abdestillirt, der Rückstand mit $50 \mathrm{cc}$ Natronhydratlösung (etwa 4 Aequivalente Natronhydrat auf $1 l$ Wasser enthaltend) versetzt und der Destillation im Dampfstrome unterworfen. Das Destillat wird in Partien von je $100 c c$ gesondert aufgefangen und titrirt.

Bei der ersten Serie meiner diesfälligen analytischen Arbeiten war ich bemüht, diese Methode bis in's kleinste Detail genau einzuhalten. 
Ich benutzte hierbei die von $\mathrm{K}$ issling (diese Zeitschrift 21, 7丂5) beschriebenen Apparate. Zur Extracion diente der Soxhlet'sche Apparat, dessen Kühler ich mit Eiswasser speiste. Als Indicator benutzte ich statt der Rosolsäure eine sehr empfindliche Lackmustinctur, da ich mich durch wiederholte Versuche überzeugte, dass ich mit diesem Indicator den Endpunkt der Titrirung mit einer grösseren Präcision zu bestimmen im Stande war. Zur Titrirung benutzte ich 1/3-NormalSchwefelsäure, die also im Liter $13,33 \mathrm{~g}$ Schwefelsäure $\left(\mathrm{SO}_{3}=40\right)$ enthielt; es entsprach demnach je $1 c c$ dieser Säure $0,054 \mathrm{~g}$ Nicotin. Damit bezüglich meiner Probeflüssigkeit jeder Zweifel völlig ausgeschlossen sei, bereitete ich mir eine Nicotinlösung, die auf $100 c c$ genau $2 \mathrm{~g}$ gelöst enthielt. Die Probe ergab, dass je $10 c c$ dieser Lösung $3,7 c c$ meiner Säure zur Sättigung brauchten; daher $0,054 \times 3,7=0,1998 \mathrm{~g}$ Nicotin; ich fand also auf je 100 Theile meiner Nicotinlösung statt 2,0 blos $1,998 \mathrm{~g}$ Nicotin, d. i. minus $2 \mathrm{mg}$, was wohl der dem Nicotin anhaftenden hygroskopischen Feuchtigkeit zuzuschreiben ist.

Bei meinen nach Kosutány's Methode ausgeführten Parallelarbeiten war ich ebenfalls bemüht, mich strenge an seine Vorschrift zu halten, nur benutzte ich statt dem Mischcylinder markirte Flaschen mit engem Halse und gut schliessendem Korke; es gelang mir hierbei, die Flüssigkeiten so innig zu mengen, dass die Berührung des wässrigen Nicotinanszuges mit dem Petroleumäther nichts zu wünschen übrig liess, und ein Verlust durch Verspritzen ganz ausgeschlossen war. Zur Titrirung benutate ich die oben erwähnte $1 / 3$-Normal-Schwefelsäure und zum Zurücktitriren der frei gebliebenen Schwefelsäure statt Barythydrats $1 / 3$-NormalNatronlauge.

Ich erhielt hierbei nebenstehende Resultate (siehe Tabelle S. 281).

Bezüglich der in dieser Tabelle angeführten analytischen Daten erlaube ich mir noch Folgendes zu bemerken:

1. $\gg$ Jungferntabak $\ll$ wurde hierzulande bis zur jüngsten Zeit ein solcher Tabak genannt, den der Producent vor oder nach dem Gährungsprocesse trotz der Wachsamkeit der Finanzorgane gesetzwidrig heimlich verkaufte; es war dies kurz gesagt ein geschmuggelter Tabak, der keine Beize erhielt, noch anderweitig fabrikmässig behandelt wurde. In neuerer Zeit fand sich aber das königliche Finanzärar - auf allgemeines Verlangen - genöthigt, auch einen solchen Rohtabak in den Handel zu bringen. Die zu dieser analytischen Arbeit angewendeten 
Vedrödi: Analyse des Tabakes und seiner Fabrikate.

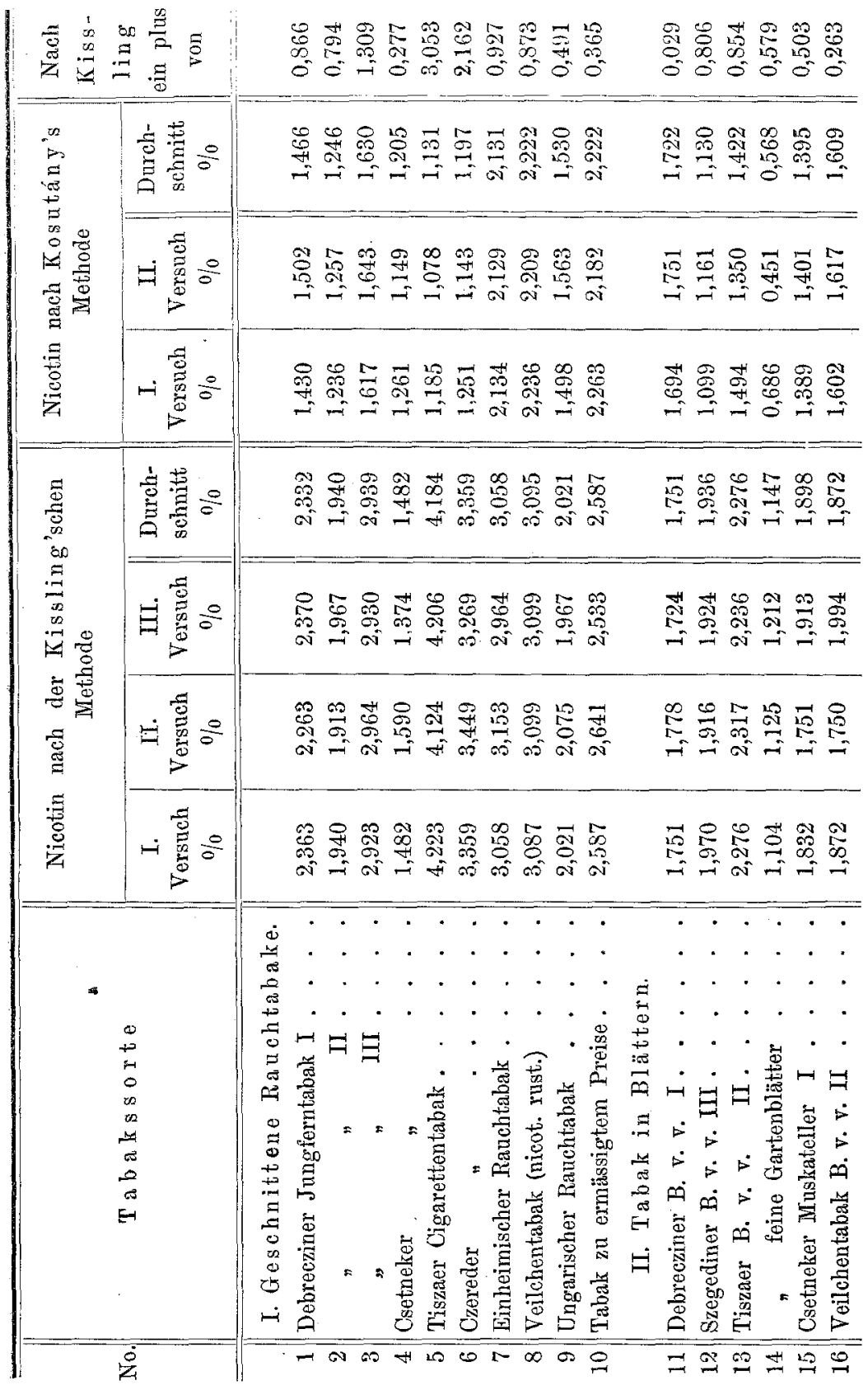


Tabakssorten erhielt ich auf eigenes Ausuchen in Folge eines königlich ungarischen Finanz-Ministerial-Rescriptes von der hiesigen Tabaksfabrik.

2. Bei meinen Analysen nach $\mathrm{K}$ issling's Methode benutzte ich zur Extraction des Nicotins aus dem mit der alkoholischen Natronlösung befeuchteten Tabake einen Soxhlet'schen Extractions-Apparat, dessen Rückflusskühler $18 \mathrm{~cm}$ lang war, bei einem Durchmesser von $31_{2} \mathrm{~cm}$.

3. Zum Abdestilliren des Aethers und später des Nicotins benutzte ich im Anfange mit Vorliebe die von Kissling empfohlenen Kolben von $400 \mathrm{cc}$ Inhalt mit kurzem Halse; später überzeugte ich mich, dass diese kurzhalsigen Kolben während der Destillation das Uebersteigen der zum Schäumen sehr geneigten alkalischen Flüssigkeit ungemein erleichtern. Ich benutzte daher später - wie Kissling dieses auch selbst in seiner zweiten Abhandlung (diese Zeitschrift 22, 211) anräth - nur Rundkolben mit $16 \mathrm{~cm}$ Halslänge, deren Hals einen Durchmesser von $3^{1} / 2 \mathrm{~cm}$ hatte. Selbst bei diesen Kolben leistete mir ein kleines in den Destillirkolben geworfenes Paraffinstücklein gute Dienste, um das Schäumen zu verhüten.

Um die Condensation des eingeleiteten Wasserdampfstromes zu verhindern, stellte ich den Destillirkolben bis zum Halse in ein Sandbad and leitete den Wasserdampf erst ein, als die Destillation schon begann. Bei diesem Verfahren hatte ich immer nur eine kleine Menge wässriger Flüssigkeit im Kolben.

4. Nach Kissling's ursprünglichem Verfahren wird der mit der alkoholischen Natronlösung befeuchtete Tabak nach sorgfältigem Umrühren in eine Papierdüte geschüttet und mit Aether extrahirt. Die auf diese Weise ausgefuhhrten Nicotinbestimmungen zeigen den nach der Kosutány'schen Methode ausgeführten gegenüber - wie dies auch aus meiner Tabelle ersichtlich ist - immer höhere Werthe für Nicotin und zwar bei schnellerem Arbeiten höhere, bei langsamerem geringere Werthe.

Besonders auffallend war die Differenz, wenn ich den Tabak nach dem Zusammenbringen mit der alkoholischen Natronlösung 1 bis 2 Tage bei gewöhnlicher Zimmertemperatur stehen liess, wobei ich immer weniger Nicotin erhielt. Ich dachte daher an die Möglichkeit der Verflüchtigung dieses Stoffes; meine späteren Controlversucbe überzeugten mich jedoch von der Unrichtigkeit dieser Annahme. 
Der Tabak enthält nämlich ausser Nicotin auch Ammoniak im gebundenen Zustande, wahrscheinlich an organische Säuren gebunden. Die Aufgabe der alkoholischen Natronlösung ist hier eine doppelte; einerseits die Isolirung des Nicotins von den Säuren, an welche dasselbe gebunden ist, andererseits das Freimachen des Ammoniaks. Je schneller man das angefeuchtete Tabakspulver nach dem Mischen mit dieser Lösung in die Papierdüte und zur Extraction bringt, desto weniger Zeit hat das Ammoniak zur Verfüchtigung; je langsamer man diese Arbeit vollzieht und je länger man das Natron auf den Tabak einwirken lässt, desto vollständiger wird das Ammoniak ausgetrieben und desto mehr stimmen die Resultate mit den nach der Kosutány'schen Methode ausgeführten überein. Schon nach 12-stündigem Stehen in einer offenen Reibschale wird das so befeuchtete Tabakspulver ganz trocken, verliert seinen starken Ammoniakgeruch und die Resultate zeigen eine grössere Uebereinstimmung, wie dieses in meiner Tabelle beim Tabak No. $11 \mathrm{zu}$ sehen ist, wo die Differenz zwischen beiden Analysen bloss $0,029 \%$ beträgt.

Um den Einfluss, den das längere Stehenlassen auf die Resultate ausübt, genauer zu studiren, habe ich in drei Partien je $20 \mathrm{~g}$ Tabak abgewogen and mit $10 \mathrm{cc}$ alkoholischer Natronlösung befeuchtet; von diesen wurde die Probe I schon nach 12 Stunden mit Aether im Extractions-Apparate extrahirt, während die anderen zwei Proben alle 24 Stuuden einmal mit $10 \mathrm{cc}$ dieser Natronlösung befenchtet wurden; die Probe II wurde nach 2 Tagen, die Probe III erst nach 3 Tagen mit Aether extrahirt.

Um die Untersuchung dieser Proben nach zwei Methoden möglich zu machen, brachte ich den ätherischen Auszug genau auf $200 \mathrm{cc}$ und theilte ihn in zwei Theile zu je $100 c c$, wovon ich $100 c c$ nach Kissling weiter untersuchte, die anderen $100 c c$ mit ebenso viel destillirtem Wasser mischte, den Aether anf dem Wasserbade verdunsten liess und auf diese Weise das Nicotin wieder in eine wässrige Lösung überführte, welch letztere ich nach dem Ablühlen mit Petroläther mischte, schüttelte und weiter ganz nach Kosutány's Methode behandelte. Ich erhielt hierbei folgende Resultate:

Bei der Probe I nach Kissling 2,16\%, nach Kosutány 1,68\% Nicotin.

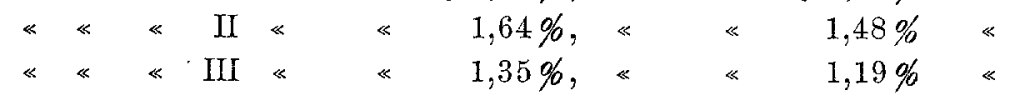


Aus diesen Proben ersehen wir, dass ich weder nach Kissling, noch nach Kosutány ganz constante Werthe erhielt; die Differenz zwischen beiden Methoden war bei der Probe I 0,48\%, bei der Probe II $0,16 \%$, bei der Probe III 0,16\%. Diese Differenz lässt sich nur durch die voliständigere Verfüchtigung des Ammoniaks oder durch die Verflüchtigung oder Zersetzung des Nicotins erklären.

Um mich zu überzeugen, $o b$ sich das Nicotin beim längeren Stehenlassen des mit der Natronlösung befeuchteten Tabakes nicht etwa verflüchtigt oder zersetzt, machte ich drei Proben mit reiner Nicotinlösung. $\mathrm{Zu}$ jeder Probe maass ich $10 \mathrm{cc}$ Nicotinlösung ab, enthaltend $0,2 \%$ Nicotin. Jede Portion mischte ich mit $10 \mathrm{cc}$ alkoholischer Natronlösung und liess sie alle in offenen Gefässen bei gewöhnlicher Zimmertemperatur stehen. Dann bestimmte ich nach Kissling durch Destillation im Wasserdampfstrome unter Anwendung von 50 cc Natronhydrat das Nicotin und zwar bei Probe I nach 12 stündigem, bei Probe II nach zweitägigem und bei Probe III nach dreitägigem Stehen. Ich erlielt:

Bei der Probe I . . . . 0,998g Nicotin.

$\ll \ll \ll$ II . . . $0,987 \mathrm{~g} \ll$

$\ll \ll \quad$ III . . . . $0,969 g \ll$

Hieraus ist zu ersehen, dass das 2-3tägige Stehenlassen die Resultate durchans nicht beeinflusst, denn die hier beobachtete kleine Differenz ist wohl als ein kaum nennenswerther minimaler Beobachtungsfehler anzusehen.

Aus diesen Resultaten schliesse ich, dass die beim längeren Stehenlassen des mit der Natronlösung befeuchteten Tabakspulvers beobachtete und oben angeführte Differenz ausschliesslich der vollständigeren Verfüchtigung des Ammons zugeschrieben werden kann.

5. Nach dem Befeuchten mit alkoholischer Natronlösung lässt Kissling das feuchte Tabakspulver in einer Papierdüte im Extractions-Apparate mit Aether extrahiren.

Obwohl ich bezüglich der vollkommenen Extrahirbarkeit des Nicotins zu zweifeln gar keinen Grund hatte, prüfte ich dennoch auch diesen Theil der Methode. Ich unterwarf den mit Aether extrahirten Tabaksrückstand einer Destillation im Wasserdampfstrome, und zwar wurden in drei Partien folgende Versuche ausgeführt:

I. Der extrahirte Rüclsstand von $20 \mathrm{~g}$ Tabak wurde mit $100-150 \mathrm{cc}$ Wasser und $50 \mathrm{cc}$ der Natronlösung von Kissling (4 Aeq. Natronhydrat auf 17 Wasser) - 
II. Dieselbe Menge vom extrahirten Tabake mit 100-150 cc Wasser und $50 \mathrm{cc}$ Kallimilch -

III. Dieselbe Menge vom extrahirten Tabake bloss mit $150-200 c c$ Wasser ohne jede Zuthat

im Wasserdampfstrome so lange destillirt, bis ich von einer jeden Probe $400-500$ co Destillat erlielt.

Das von den einzelnen Proben erhaltene Destillat wurde in zwei Theile getheilt und ein Theil hiervon mit Kaliumquecksilberjodid auf Nicotin, ein anderer Theil mit Lackmus und, nach dem Ansäuern mit Schwefelsäure und Eindampfen, mit Natronlauge und in verdünnte Salzsäure eingetauchtem und darüber gehaltenem Glasstabe auf Ammoniak geprüft.

Hierbei wurden folgende Resultate gewonnen:

I. Bei der Probe I erhielt ich ein Destillat, welches die violette Lackmustinctur blau färbte, aber mit Kaliumquecksilberjodid keine Nicotinreaction gab. Die Bläuung konnte nur durch Ammoniak hervorgerufen worden sein.

II. Bei der Probe II erhielt ich ein Destillat, welches die Farbe der violetten Lackmustinctur nicht änderte, aber mit Kaliumquecksilberjodid eine Trübung gab.

III. Bei der Probe III erhielt ich ein Destillat, welches die Lackmustinctur unverändert liess und auch mit Kaliumquecksilberjodid keine Trübung gab.

Das Destillat von I wurde mit verdünnter Schwefelsäure angesäuert, dann auf dem Wasserbade zur Trockne eingedampft, der Rückstand mit Natronlauge befeuchtet. Es wurde hierhei sowohl durch den Geruch, als auch mittelst eines in verdünnte Salzsäure eingetauchten und über die Schale gehaltenen Glasstabes das frei werdende Ammoniak constatirt.

Durch diese Versuche ist erwiesen, dass sich bei der Destillation des Tabaksrückstandes kein Nicotin ergab, dass also die Extraction nach Kissling's Methode eine vollständige ist. Die Bläuung des Destillates I konnte nur auf Rechnung des Ammoniaks geschrieben werden, welches sich bei der Destillation im Wasserdampfstrome aus den stickstoffhaltigen Bestandtheilen des extrahirten Tabakes unter Einwirkung der Natronlauge bildete.

Die Bildung des Ammoniaks aus den stickstoffhaltigen Bestandtheilen der organischen Stoffe des Tabakes 
unter Einwirkung der Natronlauge und bei Benutzung des Wasserdampfstromes ist jedenfalls eine sehr beachtenswerthe Erscheinung, und es drängt sich hierbei die Frage auf, ob man diese Erscheinung nicht auch benutzen könnte zur Bestimmung des Stickstoffes respective des Proteïs in organischen Substanzen statt der Schwefelsäure nach der Kjeldahl'schen Methode.

Die Ursache der Trübung des Destillates durch Kaliumquecksilberjodid beim Versuche II ist nicht ganz aufgeklärt, so viel ist jedoch sicher, dass diese Erscheinung nicht vom Nicotin hervorgerufen wurde, weil das Destillat nicht alkalisch reagirte. Meine eigens angestellten Versuche zeigten ganz unzweideutig, dass eine solche Trübung auch bei der Destillation der reinen Kalkmilch im Wasserdampfstrome bei gänzlichem Ausschlusse des Tabaksrückstandes ganz deutlich entstand.

6. Die nach Punkt 5 erhaltene ätherische Lösung wird jetzt nach Kissling durch Destillation vom überschüssigen Aether befreit und hierauf das Nicotin im Wasserdampfstrome unter Zusatz von 50 cc Natronlösung abdestillirt und titrirt.

Zur Controle dieses Theiles der Methode war zu ermitteln:

I. Ob beim Abdestilliren des Aethers nicht auch ein Theil des Nicotins mit übergeht?

II. Ob hierbei neben dem Nicotin nicht auch ein Theil des Ammoniaks im Rückstande bleibt?

III. $O b$ sich das Nicotin mittelst des Wasserdampfstromes vollständig übertreiben lässt?

Bezüglich der Frage I behauptet Schlösing, dass das Ammoniak bei der Extraction des Tabakes vom Aether gelöst wird, aber als flüchtigerer Körper bei der Destillation des Aethers mit letzterem gleichzeitig abdestillirt wird. Meine diesbezunglichen Versuche überzeugten mich, dass dies nur bedingungsweise zutrifft, nämlich nur in dem Falle, wenn der Aether wasserfrei und das Tabakspulver so trocken ist, dass der nach der Destillation erhaltene Rückstand ganz trocken zurückbleibt. Bei Anwendung feuchten Aethers hingegen lässt sich das Ammoniak sehr schwer vertreiben; die letzten Reste enthalten immer Ammoniak, welches später bei der Destillation mit Wasserdampf mit dem Nicotin übergeht, als solches in Berechnung kommt and ver- 
möge seines niedrigen Atomgewichtes im Vergleiche zum hohen Atomgewichte des Nicotins schon bei geringer Menge die Resultate bedeutend erhöht.

Um das Verhalten des Nicotins und des Ammoniaks beim Destilliren mit Aether zu studiren, machte ich zwei Proben. Bei der ersten mischte ich $10 c c$ Nicotinlösung (enthaltend 0,945\% Nicotin) mit $200 c c$ Aether; bei der zweiten eben so viel von derselben Nicotinlösung mit $200 \mathrm{cc}$ Aether und $1 c c$ Salmiakgeist (enthaltend 2,106\% Ammon), destillirte den Aether $a b$ und titrirte einerseits je eine Probe der eben angeführten Mischungen vor der Destillation und je eine Probe nach erfolgter Destillation, wobei ich die erhaltenen Rückstände titrirte.

\section{Probe I.}

$10 c c$ Nicotinlösung erforderten vor der Destillation $1,75 c c$ Schwefelsäure. Der Rückstand erforderte nach der Destillation . 1,50 *

Also minus . $0,25 c c$ Schwefelsäure.

Dies entspricht $0,135 \%$ Nicotin, welches sich theils mit dem Aether verflüchtigte, theils zersetzte.

\section{Probe II.}

Hier verwendete ich dieselbe Lösung. Es entsprachen:

$10 \mathrm{cc}$ Nicotinlösung vor der Destillation . . = 1,75 cc Schwefelsäure. $\begin{aligned} 1 \ll \text { Salmiakgeist } * * \quad * \quad & =35,10 * \\ \text { Zusammen } & .=36,85 c c \text { Schwefelsäure. }\end{aligned}$

Nach der Destillation waren erforderlich zur

Titrirung des Rückstandes . . . . . $=20,50 \ll \quad$ «

Hiervon abgezogen die zur Titrirung der $10 c c$

Nicotinlösung erforderlichen Cubikcenti-

meter Schwefelsäure laut Probe I . . = 1,50* *

Für das im Rückstande gebliebene Ammon

waren somit erforderlich . . . . . $=19,00 c c$ Schwefelsäure.

$1 c c$ meiner $1 / \mathrm{g}$-Normal-Schwefelsäure entsprach $0,054 g$ Nicotin oder $0,006 \mathrm{~g}$ Ammonium $\left(\mathrm{NH}_{4}=18\right)$; die $19 c c$ Schwefelsäure entsprachen demnach $0,114 \mathrm{~g}$ Ammonium.

Diese Versuche machen es zweifellos:

1. Dass sich bei der Destillation mit Aether auch etwas Nicotin verflüchtigt. 
2. Dass sich das Ammon mit dem Aether bei der Destillation nicht ganz verflüchtigt, sondern dass ein kleiner Theil im Destillationsrückstande zurückbleibt und auf die Resultate sehr störend einwirkt; und zwar um so mehr, je feuchter der Aether ist, den man zur Extraction des Tabaks verwendet, und je mehr Ammoniak der Tabak enthält.

Es ist hierbei selbstverständlich, dass dieser Fehler um so mehr vermieden werden kann, je trockener der zu untersuchende Tabak ist, und je weniger Fenchtigkeit der Aether enthält. Hiermit wären auch die unter diesem Punkte aufgeworfenen Fragen I und II erledigt.

Es wäre nur noch die Frage III zu beantworten, nämlich ob sich das Nicotin mittelst des Wasserdampfstromes vollständig überdestilliren lässt? Un diese Frage beantworten zu können, destillirte ich $10 c c$ meiner 2 procentigen Nicotinlösung nach dem Mischen mit $50 c e$ Natronhydratlösung mit dem Wasserdampfstrome. Vom Destillate wurden $500 c c$ aufgefangen und in Partien von je $100 c c$ titrirt. Das Destillat erforderte im Ganzen 3,4 ce $1 / 3$-Normal-Schwefelsäure. Ein zweiter und dritter ähnlicher Versuch ergaben beinahe dasselbe Resultat, die Differenz war sehr gering. 3,4cc Schwefelsäure entsprechen $0,1836 \mathrm{~g}$ Nicotin (1 cc Schwefelsäure $=0,054 \times 3,4=0,1836 \mathrm{~g}$ Nicotin $=1,836 \%$.

Durch diese Versuche ist daher constatirt, dass das Nicotin mittelst des Wasserdampfstromes sich beinahe vollständig abdestilliren lässt, und dass die kleine Differenz auf circa $0,1-0,20 \%$ zu schätzen ist.

Wie bei der Methode von Kissling, war ich auch bemüht bei der Methode Kosutány's die eventuellen Fehlerquellen auszuforschen. Es mussten folgende Fragen aufgestellt werden:

I. Ob sich durch Behandeln mit Kalkmilch binnen 2-3 Tagen das sämmtliche Ammon ans dem Tabakspulver austreiben lässt?

II. $\mathrm{Ob}$ sich hierbei nicht auch etwas vom frei werdenden Nicotin verflüchtigt?

III. Ob sich das mit Kalkmilch frei gemachte Nicotin aus $20 \mathrm{~g}$ Tabakspulver mittelst $300-400 c c$ Wasser auf dem Trichter filtrirt vollständig aussüssen lässt?

IV. Ob das im wässrigen Tabalssauszuge gelöste Nicotin, mit Petroläther geschüttelt, von letzterem vollständig aufgenommen wird?

V. Ob nach dem Ansäuern der ätherischen Nicotinlösung mit Schwefelsäure das Nicotin wieder vollständig in die wässrige Flüssigkeit übergeht? 
I. Zur Prüfung der sub I gestellten Frage mischte ich mehrere Proben Tabakspulver zu je $20 \mathrm{~g}$ mit $10-15 \mathrm{cc}$ Kalkmilch, liess das Gemenge in einer Schale bei gewöhnlicher Zimmertemperatur in einem Exsiccator stehen, dessen Schale mit concentrirter Schwefelsäure gefüllt war, und befeuchtete die erste Probe nach 2 Tagen, die zweite nach 3 Tagen, und die dritte nach 4 Tagen mit $10 c c$ der alkoholischen Natronlösung von Kissling. Die Proben warden im Tage öfters umgerührt, sie trockneten aus, verloren ihren Ammongeruch und zeigten schliesslich nur einen Geruch nach Nicotin. Nach 3-4 Tagen konnte das Ammon nach Befeuchten mit der Natronlösung Kissling's weder durch "den Geruch erkannt werden, noch mittelst des in verdünnte Salzsäure eingetauchten und über die Probe gehaltenen Glasstabes. Ein Beweis, dass sich das Ammon auf diese Weise vollständig entfernen lässt.

II. Zur Prüfung der Frage II machte ich vier Proben. Durch die ersten zwei wollte ich das Verhalten des reinen Nicotins, durch die anderen zwei hingegen das Verhalten des mit Ammonsalzen gemengten Nicotins prüfen. Ich goss in vier Partien genau je $10 c c$ meiner 2 procentigen Nicotinlösung (enthaltend $0,2 g$ Nicotin) in vier gleich weite Porzellanschalen, mengte zwei hiervon mit $2 g$ Salmiak, übergoss alle vier Proben mit je $10 \mathrm{cc}$ der alkoholischen Natronlösung Kissling's und destillirte nach"Zusatz von $50 c c$ Natronlösung (4 Aeq. Natronlösung auf 1 Liter Wasser) im Wasserdampfstrome, und zwar wurden die zwei ersten Proben, welche kein Ammonsalz zugemengt erhielten, nach zwei, die anderen Proben nach vier Tagen destillirt und die Destillate titrirt.

Zur Titrirung aller vier Destillate verbrauchte ich $3,7 \mathrm{cc}$ meiner $1 / 3$-Normal-Schwefelsäure, entsprechend $0,1998 \mathrm{~g}$ Nicotin. Diese Versuche zeigen ganz evident, dass man den mit Kalkmilch befeuchteten Tabak ganz ruhig 3-4 Tage in einem offenen Gefässe stehen lassen kann, ohne hierdurch einen Verlust an Nicotin befürchten zu müssen.

III. Zur Prüfung der Frage III füllte ich den auf dem Filter befindlichen noch feuchten Tabaks-Rückstand in einen Rundkolben, goss $100 c c$ Wasser und $50 c c \mathrm{~K}$ is sling 'sche Natronlauge hinzu, und destillirte hiervon im Wasserdampfstrome $500 \mathrm{cc} a \mathrm{~b}$. Das Destillat wurde mit violetter Lackmustinctur b lau und gab mit Kaliumquecksilberjodid keine Trübung, es enthielt somit kein Nicotin. Ich habe bereits im Verlaufe dieser Abhandlung gezeigt, dass unter der 
Einwirkung der Natronlauge auf die stickstoffhaltigen organischen Bestandtheile des Tabakes Ammoniak entsteht, welches auch hier die Blaufärbung des Lackmus veranlasste.

IV. Zur Prüfung der Frage IV machte ich zuerst folgenden Versuch: Ich säuerte den mit Petroläther extrahirten Tabaks-Auszug mit verdünnter Schwefelsäure an, um das eventuell darin befindliche Nicotin zu binden, trocknete ihn im Wasserbade bis auf einen kleinen Rest von $50-60 c c$, brachte den 'so eingedickten Extract in einen Destillirkolben, goss $50 c c$ von der Natronlösung Kissling's hinzu, um das Nicotin wieder frei zu machen, und destillirte die auf diese Weise alkalisch gemachte Flüssigkeit im Wasserdampfstrome; ich erhielt fractionirt $500 \mathrm{cc}$ Destillat. Dasselbe färbte die violette Lackmustinctur blan und gab mit Kaliumquecksilberjodid keine Nicotinreaction. Die Blaufärbung verursachte auch hier das Ammoniak.

Aus diesem Versuch wollte ich schliessen, dass der wässrige Extract kein Nicotin enthalte. Eine zweite Probe überzeugte mich jedoch von der Unbrauchbarkeit dieses Verfahrens, Ich mischte nämlich $10 \mathrm{cc}$ von meiner 2 procentigen reinen Nicotinlösung mit $500 \mathrm{cc}$ Wasser, titrirte diese Flüssigkeit mit meiner $1 / 3$-Normal-Schwefelsänre, dampfte die so erhaltene Flüssigkeit im Wasserbade bis auf 50-60 cc ein, goss in den Destillirkolben und mischte daselbst mit $50 \mathrm{cc}$ Natronhydratlösung. Die Flüssigkeit wurde hierdurch wieder stark alkalisch, und das Nicotin sollte sich in ihr wieder im isolirten Zustande befinden. Ich destillirte daher im Wasserdampfstrome und sollte im Destillate das Nicotin wieder erhalten. Allein das Destillat wurde mit violetter Lackmustinctur nicht blau und gab mit Kaliumquecksilberjodid keine Reaction auf Nicotin. Ein Beweis, dass sich das Nicotin als schwefelsaures Salz eingedampft vollständig zersetzt, und dass diese Methode zur Bestimmung des Nicotins ganz unbrauchbar ist.

Um mich daher zu überzeugen, ob das mit Petroläther behandelte wässrige Extract noch Nicotin enthält, war ich genöthigt, in einer neuen Probe dieses Extract selbst, mit $50 c c$ Natronhydratlösung gemischt, zu destilliren. Ich benutzte hierbei keinen Wasserdampfstrom, so lange im Destillirkolben die Flüssigkeit in genügender Menge vorhanden war; ich destillirte und untersuchte fractionirt, zu je $100 c c$, die Destillate, von welchen ich uber $800 c c$ abtröpfeln liess. Ich fand, dass sich die sämmtlichen Destillate mit violettem Lackmus bläuten, und selbst der letzte Rest des Destillates mit Kaliumquecksilberjodid eine Trübung gab. 
Da weder das nach $K$ issling mit Aether extrahirte, noch das nach Kosutány mit Wasser ausgesüsste Tabakspulver auf diese Weise behandelt mit Kaliumquecksilberjodid eine Nicotinreaction gab, musste ich diese Trübung als Kennzeichen der Anwesenheit des Nicotins ansehen und fühle mich berechtigt, dieses als eine Fehlerquelle der Methode von Kosutány zu bezeichnen.

V. Zur Prüfung der Frage V musste ich noch constatiren, $a b$ aus der ätherischen Nicotinlösung durch Ansäuern mit Schwefelsäure das sämmtliche Nicotin in die wässrige Lösung übergeht? Ich goss in den von der angesäuerten Lösung getrennten Aether $200 c c$ destillirtes Wasser, färbte mit violetter Lackmustinctur, mischte die Flüssigkeiten durch starkes Schütteln, liess sie ruhen, trennte die wässrige Flüssigkeit vom Petroläther und wiederholte dieses Ausspülen noch zweimal. Ich fand hierbei, dass während bei der Trennung der ersten wässrigen Flüssigkeit, welche das schwefelsaure Nicotin enthielt, diese Flüssigkeit schwach zwiebelroth war; das erste auf den Petroläther neuerdings aufgegossene Spülwasser kaum mehr ein wenig geröthet wurde, die übrigen Spülwasser hingegen ausnahmslos violett blieben. Ein Beweis, dass die freie Schwefelsäure schon bei der ersten Trennung vollständig abgeschieden wurde. Ich destillirte den so gereinigten Petroläther bis auf einen kleinen Rückstand, goss zu diesem Rückstande $50 \mathrm{cc}$ von der Kissling'schen Natronlösung und destillirte weiter im Wasserdampfstrome. Das Destillat blieb mit Lackmus violett und gab mit Kaliumquecksilberjodid keine Nicotinreaction; ein Beweis, dass es kein Nicotin enthielt, und dass die Trennung des Nicotins nach dieser Methode vollständig vor sich geht. $\mathrm{Da}$ bei dieser Destillation in der zu destillirenden Flüssigkeit keine freie Schwefelsäure vorhanden war, ist die Befürchtung einer Zersetzung des Nicotins ansgeschlossen.

Um mich hiervon zu überzengen, neutralisirte ich $5 c c$ Nicotinlösung (enthaltend $0,0486 \mathrm{~g}$ Nicotin) mit $0,9 c c$ meiner $1 / 3$ - NormalSchwefelsäure, mischte mit $100 \mathrm{cc}$ Petroläther, destillirte den Aether $\mathrm{ab}$, mischte den Rückstand mit $25 \mathrm{cc}$ Natronlauge und destillirte im Wasserdampfstrome. Vom Destillat wurde $1 c c$ mit Kaliumquecksilberjodid auf Nicotin geprüft, der übrige Theil mit Lackmustinctur versetzt und titrirt. Das Kaliumquecksilberjodid zeigte hierbei eine Trübung, ein Beweis des Vorhandenseins von unzersetztem Nicotin. Zur Titri- 
rung waren $0,8 c c 1 / 3$-Normal-Schwefelsäure erforderlich. Es entspricht dieses $0,0432 \mathrm{~g}$ Nicotin.

Gestützt auf die hier angeführten analytischen Arbeiten, sei es mir erlaubt über den Werth der beiden von mir besprochenen Methoden einen Vergleich anzustellen und ein objectives Urtheil abzugeben. Dieses lautet dahin, dass keine dieser Methoden absolut fehlerfrei ist. Die Resultate müssen nach Kissling immer zu hoch, nach Kosutány immer zu nieder ausfallen. Bei Anwendung der Methode von Kissling wird man nur dann im Stande sein, Resultate zu erzielen, welche der Wahrheit näher kommen, wenn man auf die Entfernung des Ammoniaks vor der Extraction mit Aether eine grössere Sorgfalt verwendet. Dies liesse sich dadurch erreichen, dass man das Tabakspulver nach Befeuchten mit der alkoholischen Natronlösung unter öfterem Umrühren 3-4 Tage hindurch über Schwefelsäure im Exsiccator stehen liesse, und nach dem Austrocknen alle 24 Stunden 1-2 mal mit verdünntem Alkohol befeuchtete, und zwar so lange, bis man sich überzengt, dass der Tabak kein Ammoniak mehr enthält. Es müsste ferner zur Extraction des Tabakspulvers aus Vorsicht ein möglichst wasserfreier Aether verwendet werden. Unter diesen Bedingungen dürfte es gelingen, nach Destillation des Aethers einen Rückstand zu erhalten, der kein Ammoniak enthält. Diese Methode kann jedoch selbst bei Einhaltung dieser Bedingungen keine absolut guten Resultate liefern, weil beim Befeuchten des Tabakspulvers mit der Natronlösung ein Theil der im Tabake befindlichen stickstoffhaltigen organischen Stoffe löslich wird, in die ätherische Lösung übergeht und beim Abdestilliren des Aethers im Rückstande zurückbleibt, hier unter Einwirkung des Natronhydrates und des Wasserdampfstromes sich in Ammoniak verwandelt, welches bei der Destillation mit dem Nicotin abdestillirt, titrirt wird, und fälschlich als Nicotin zur Berechnung kommt.

Die Methode von Kosutány leidet an dem entgegengesetzten Fehler, indem bei Anwendung derselben - wie ich dies bereits gezeigt habe - ein kleiner Theil des Nicotins in dem wässrigen Tabaksextracte zurückbleibt und sich der Bestimmung entzieht. Es bleibt eine offene Frage, ob man durch öfteres Ausschütteln mit Petroläther oder eventuell durch Benutzung einer grösseren Menge von Petroläther im Stande sein wird, diesem Extracte das sämmtliche Nicotin zu entziehen und in eine bestimmbare Form zu bringen. Gelingt dieses, so ist diese Methode 
vermöge ihrer Einfachheit, Bequemlichkeit and Sicherheit in der Ausführung sehr werthvoll und sehr beachtenswerth.

Eben so bedeutend wie die Tabakfabrikation ist die Cigarrenfabrikation in Ungarn; es wird hierzu theils ausländischer, theils inländischer Tabak verwendet. Laut statistischen Nachweisen des königlich angarischen Finanzministeriums wurden im Jahre 1891 von ausländjschen Tabaksorten Havannah, Java, Sumatra, Brasil, Cuba, Manilla, Ohio, Virginia $\boldsymbol{u}$. s. w. im Ganzen $3,899,626 \mathrm{~kg}$ im Werthe von $4,739,483 \mathrm{fl}$. und $73 \mathrm{kr}$. österreichischer Währung eingekauft. Hierzu kommen noch die fertig angekauften Fabrikate, und zwar $93,248 \mathrm{~kg}$ Schnupftabak und $1,878,275$ Stück ächte Havannah-Cigarren und Special-Cigarren im Werthe von 256,933 fl. $61 \mathrm{kr}$.

Im gewöhnlichen Trafikverschleisse finden wir die in der folgenden Tabelle angeführten Cigarrensorten, und zwar:

\begin{tabular}{|c|c|c|c|c|c|c|c|c|c|}
\hline$\dot{z_{i}}$ & 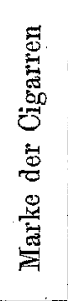 & $\begin{array}{c}\text { Name } \\
\text { der } \\
\text { Cigarren }\end{array}$ & 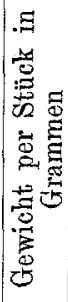 & 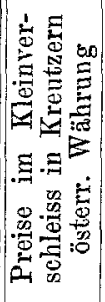 & $\begin{array}{r}\text { Dn } \\
\text { schnit } \\
\text { Nicotir } \\
\text { d } \\
\text { verwe } \\
\text { Tabake } \\
\text { Kiss- } \\
\text { ling } \\
0 \%\end{array}$ & $\begin{array}{l}\text { ch- } \\
\text { licher } \\
\text { gehalt } \\
\text { s } \\
\text { deten } \\
\text { nach } \\
\text { Kosu- } \\
\text { tány } \\
\%\end{array}$ & $\begin{array}{l}\text { Durch } \\
\text { licher } \\
\text { gehalt } \\
\text { Cig } \\
\text { in Gr }\end{array}$ & $\begin{array}{l}\text { hnitt- } \\
\text { cotin- } \\
\text { einer } \\
\text { re } \\
\text { nmen } \\
b\end{array}$ & $\begin{array}{c}\text { Fabricirt } \\
\text { warden hier- } \\
\text { ron im } \\
\text { Jahre } 1891 \\
\text { Stück }\end{array}$ \\
\hline 1 & $\mathbf{A}-\mathbf{A}$ & egalitas. & 6,09 & 9,0 & 2,215 & 1,614 & 0,162 & 0,149 & $4,946,798$ \\
\hline 2 & A & Trabuco. & 5,51 & 8,0 & 2,023 & 1,348 & 0,113 & 0,076 & 16,4 \\
\hline 3 & B-B & Britanica. . & 5,98 & 7,0 & 1,865 & 1,242 & 0,104 & 0,068 & $39,316,055$ \\
\hline 4 & $\mathrm{~B}$ & Milares . . & 6,83 & 6,5 & 1,736 & 1,156 & 0,118 & 0,078 & $12,801,000$ \\
\hline 5 & $\mathrm{C}$ & Panatelas & 4,86 & 6,5 & 1,718 & & & & 450,205 \\
\hline 6 & D & Cuba & 6,70 & 6,0 & 2,127 & 1,418 & 0,145 & 0,096 & 366 \\
\hline 7 & $\mathbf{E}$ & Cuba-Portorico. & 5,78 & 5,0 & 1,968 & 1,312 & 0,114 & 0,076 & 9,635 \\
\hline 8 & F & Portorico. & 4,19 & 3,5 & 1,712 & 1,140 & 0,071 & 0,046 & $30,841,989$ \\
\hline 9 & G & Virginia & 5,24 & 5,5 & 2,885 & 1,922 & 0,156 & 0,104 & $35,132,035$ \\
\hline 10 & $\mathrm{G}-\mathrm{G}$ & Verey. & 4,92 & 4,0 & 2,834 & 1,888 & 0,093 & 0,062 & 196,800 \\
\hline 11 & $G-R$ & Kurze Virginia & 3,58 & 3,5 & 2,877 & 1,918 & 0,099 & 0,066 & - \\
\hline 12 & $\mathrm{H}$ & Kurze Ausländer & 4,71 & 2,5 & 2,247 & 1,498 & 0,109 & 0,077 & $191,645,913$ \\
\hline 13 & $\mathrm{~J}$ & Ungarische & 5,97 & 2,0 & 2,182 & 1,454 & 0,126 & 0,084 & $11,541,236$ \\
\hline \multirow[t]{2}{*}{14} & \multirow[t]{2}{*}{ K } & \multirow[t]{2}{*}{ Kurze ungarische } & \multirow[t]{2}{*}{4,71} & \multirow[t]{2}{*}{1,5} & \multirow[t]{2}{*}{2,565} & \multirow[t]{2}{*}{1,710} & 0,116 & \multirow{2}{*}{$\begin{array}{c}0,076 \\
\text { men } .\end{array}$} & $19,946,800$ \\
\hline & & & & & & & Zusa & &, 958 \\
\hline
\end{tabular}

Hierzu kommen noch 13,585,810 Stück Special-Cigarren, 521,808,383 Stück gewöhnliche und 12,140,200 Stück Special-Cigaretten. 
Durch die chemische Analyse sind wir im Stande die Qualität und die Mengenverbältnisse einiger wichtigen Bestandtheile des Tabakes z erforschen; und als wichtigster Bestandtheil wird das Nicotin, nämlich das betäubende Princip des Tabakes, angesehen. Allein verbinden wir diese analytischen Daten mit Kostproben, so kommen wir gar bald zur Erkenntniss dessen, dass die Stärke, die scharfe oder milde Beschaffenheit, sowie das Aroma des Tabakes und seiner Fabrikate in keinem Verhältnisse steht zur gefundenen Nicotinmenge, und dass das ideale Endziel der chemischen Analyse - nämlich die Möglichkeit die natürliche Beschaffenheit des Tabakes in jeder Hinsicht, in analytischen Daten, in Zahlen auszudrücken - bei weitem nicht erreicht ist. Durch die Bestimmung des Nicotins allein ist ein solches Resultat auch nicht erreichbar, besonders wenn man bedenkt, dass das Nicotin im Tabake auf verschiedene Weise, nämlich an starke oder schwache Säuren, ganz oder halb gebunden, vielleicht theilweise auch im freien Zustande vorhanden sein kann, and seine Wirkung diesen Verhältnissen angemessen verschieden sein muss; ausserdem ist noch zu berücksichtigen, dass im Tabake neben dem Nicotin noch ein anderer scharfer Stoff, das Ammoniak, enthalten ist, welches als sehr flïchtige, starke Base auf die RespirationsOrgane eine trocknende, kratzende Wirkung ausübt, daher auf die Güte und auf die Beschaffenheit des Tabakes einen nicht zu unterschätzenden Einfluss ansübt, weshalb auch durch gleichzeitige Bestimmung des Ammoniaks analytische Daten gewonnen werden könnten, die uns dem idealen Ziele doch etwas näher bringen.

Ich habe, dieses berücksichtigend, die im Jahre 1892 auf dem Versuchsfelde der königl. ungarischen landwirthschaftlichen Lehranstalt in Debreczin producirten Tabake untersucht und die Ergebnisse in der folgenden Tabelle zusammengestellt.

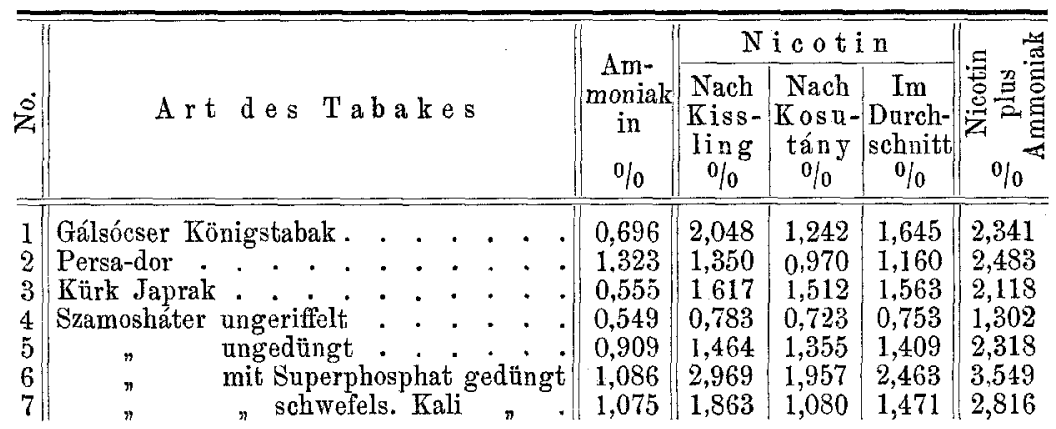


Das Ammoniak wurde hiebei nach zwei Methoden bestimmt, und zwar:

1. Nach der Methode von Kosutány wurden $20 \mathrm{~g}$ Tabak mit Kalkmilch gut angefeuchtet und unter eine Glasglocke gestellt, die zur Absorption des frei werdenden Ammoniaks in einer offenen Schale eine abgemessene Menge Normal-Schwefelsäure enthielt. Das frei gewordene Ammoniak war in 4-5 Tagen von der Schwefelsäure aufgenommen, worauf die frei gebliebene Schwefelsäure mit Normal-Natronlauge titrirt und aus der Differenz das Ammoniak berechnet wurde.

2. Die zweite Methode wurde folgenderweise ausgeführt: Ich mischte $20 \mathrm{~g}$ trockenes Tabakspulver mit $100 \mathrm{cc}$ Wasser und $100 \mathrm{cc}$ Kalkmilch und destillirte so lange im Wasserdampfstrome, als das Destillat mit Lackmus Blaufärbung zeigte. Letzteres wurde mit $1 / 3$-NormalSchwefelsäure titrirt und die verbrauchten Cubikcentimeter Schwefelsäure notirt; es bezogen sich diese a uf Nicotin + Ammoniak.

In einer zweiten und dritten Probe, wozu ich von demselben Tabake wieder $20 \mathrm{~g}$ verwendete, bestimmte ich das Nicotin, und zwar in der einen Probe nach Kissling, in der anderen nach Kosutány, und betrachtete den Durchschnitt der durch diese zwei Methoden erhaltenen Daten als dasjenige Mengenverhältniss, welches dem wirklichen Nicotingehalte am nächsten steht. Die Durchschnittszahl der zur Titrirung des Nicotins verwendeten Cubikcentimeter $1 / 3$-Normal-Schwefelsäure zog ich von der obigen auf Nicotin + Ammon bezüglichen Zahl ab and den Rest berechnete ich als Ammoniak.

Zur Benutzung dieser Methode glaubte ich durch den Umstand berechtigt zu sein, dass ich bei meinen über 250 Nicotinbestimmungen, die ich im Verlaufe dieser Arbeit ausführte, niemals Gelegenheit hatte wahrzunehmen, dass unter Einwirkung. der Kalkmilch auf die stickstoffhaltigen organischen Substanzen des Tabakes sich Ammoniak bildet. Die Daten, die ich bei Benutzung dieser Methode für Ammoniak erhielt, stimmten mit den nach Kosutány's Methode erhaltenen ziemlich überein. Ich hatte übrigens noch nicht Gelegenheit diese Methode so vielseitig und kritisch zu studiren, als ich es für nothwendig hielte, um in der Lage zu sein, sie meinen Fachgenossen empfehlen zu können.

Schliesslich muss ich noch bemerken, dass ich mir Mühe nahm das von $\mathrm{Hermstädt}$ und Trommsdorff aufgefundene und beinahe in allen ausführlicheren chemischen Lehrbüchern figurirende $\mathrm{Nicotianin}$ darzustellen, um dasselbe näher zu untersuchen. Ich wiederholte daher 
die Versuche von Hermstädt und Trommsdorff, machte Destillationsproben mit grossen und mit kleinen Mengen, benutzte sogar Eiswasser zur Kühlung und fand im Destillate das Nicotin, aber von einem beim Abkühlen krystallinischen, stearoptenartigen, indifferenten, auf der Oberfläche des Wassers schwimmenden Körper konnte ich niemals und nirgends anch nur eine Spur auffinden.

\section{Ueber die Sjöqvist'sche Methode zur Bestimmung der freien Salzsäure im Magensaft. ${ }^{1}$ ) \\ Von}

\section{Stan. Bondzyński.}

Die zahlreichen kritischen Abhandlungen über die Sjöqvist'sche Methode der Bestimmung der Salzsäure im Magensafte veranlassen mich, die Resultate der Untersuchungen mitzutheilen, die im September 1891 ausgeführt und in einer medicinischen Zeitschrift ${ }^{2}$ ) veröffentlicht wurden, weiteren chemischen Kreisen aber unbekannt geblieben. sind. Die Methode von Sjöqvist, eine von den wenigen, welche auf einer einfachen und zuverlässigen chemischen Grundlage beruhen, verdient wohl eine eingehende Besprechung. Die erwähnten Untersuchungen verfolgten den Zweck, das Verhalten des Chlorbaryums in der Glühhitze zu studiren.

Da sogar die Beständigkeit des Chlorbaryums als solchen in der Glühhitze angefochten und als Fehlerquelle bezeichnet wurde, so sollen die zunächst vorliegenden Versuche darüber Aufschluss geben. Dieselben wurden quantitativ ausgeführt. Dies geschah durch Bestimmung des Chlorgehaltes vor und nach dem Glühen. $\mathrm{Zu}$ dem $Z$ wecke wurden eine $1 / 10$-Normal-Chlorbaryumlösung und eine $1 / 10$-Normal-Silberlösung bereitet.

$30 c c$ der $1 / 10^{-N o r m a l-C h l o r b a r y u m l o ̈ s u n g ~ w u r d e n ~ m i t ~} 2 c c$ verdünnter Salpetersäure (spec. Gew. 1,2) und darauf mit $35 c c 1 / 10$-Normal-Silberlösung versetzt; der Ueberschuss der Silberlösung wurde mit Rhodanammonium unter Anwendung von Eisenammoniumalaun als Indicator nach der Volhard'schen Methode zurücktitrirt. Nachdem festgestellt war, dass $30 \mathrm{cc}$ der Chlorbaryumlösung $30 \mathrm{cc}$ Silberlösung entsprachen, wurden die Flüssigkeiten zu Versuchszwecken angewandt.

1) Die in dieser Arbeit mitgetheilten analytischen Daten wurden von Stef. Boydzyński, stud. chem., geliefert.

2) Gazeta lekarska (polnisch), Januarheft 1892. 